\title{
Going off the light-cone - a model study of quasi-GPDs
}

\author{
Shohini Bhattacharya* \\ Department of Physics, SERC, Temple University, Philadelphia, PA 19122, USA \\ E-mail: tug23108@temple.edu
}

\section{Christopher Cocuzza}

Department of Physics, SERC, Temple University, Philadelphia, PA 19122, USA

E-mail: tug83224@temple. edu

\section{Andreas Metz}

Department of Physics, SERC, Temple University, Philadelphia, PA 19122, USA

E-mail: metza@temple.edu

\begin{abstract}
Studying light-cone PDFs through Euclidean correlators is currently an active field of research. In particular, the concept of quasi parton distributions (quasi-PDFs) is at the forefront of numerical calculations of the partonic structure of strongly interacting systems using lattice QCD. Quasi-PDFs converge to their respective standard distributions if the hadron momentum goes to infinity. We investigate this quasi-distribution approach for twist-2 generalized parton distributions (GPDs) in the widely-used diquark spectator model. We illustrate analytical and numerical results of quasi-GPDs and of quasi-PDFs. We test how well the quasi-distributions agree with their standard counterparts for finite hadron momenta. Furthermore, we explore higher-twist effects associated with the parton momentum and the longitudinal momentum transfer to the target. By discussing the sensitivity of our results to model parameters, we highlight robust features of the quasi-GPDs and quasi-PDFs that one may extract from this model study. We also elaborate on a model-independent analysis of moments of quasi-distributions including relation to Ji's spin sum rule. The moment analysis suggests a preferred definition of several quasi-distributions.
\end{abstract}

Light Cone 2019 - QCD on the light cone: from hadrons to heavy ions - LC2019

16-20 September 2019

Ecole Polytechnique, Palaiseau, France

\footnotetext{
* Speaker.

$\dagger$ This work has been supported by the National Science Foundation under grant number PHY-1812359, and by the U.S. Department of Energy, Office of Science, Office of Nuclear Physics, within the framework of the TMD Topical Collaboration.
} 


\section{Introduction}

The notion of quasi parton distributions (quasi-PDFs), put forward by Ji [1], has attracted enormous attention. They are defined through purely spatial correlation functions and are thus calculable in lattice QCD. Quasi-PDFs approach their corresponding light-cone counterparts (standard PDFs) in the limit that the hadron momentum goes to infinity. Here we investigate this new approach to calculate (eight) twist-2 GPDs in a scalar diquark model (SDM) [2, 3]. In this short write-up of the talk, we mainly focus on the unpolarized quasi-GPD $H_{\mathrm{Q}}$ which corresponds to the standard GPD $H$.

\section{Definition of Quasi-GPDs}

Analogous to the standard GPDs [4], quasi-GPDs are defined through an equal-time spatial correlation function [1],

$$
F_{\mathrm{Q}}^{[\Gamma]}\left(x, \Delta ; \lambda, \lambda^{\prime} ; P^{3}\right)=\left.\frac{1}{2} \int \frac{d z^{3}}{2 \pi} e^{i k \cdot z}\left\langle p^{\prime}, \lambda^{\prime}\left|\bar{\psi}\left(-\frac{z}{2}\right) \Gamma \mathscr{W}_{\mathrm{Q}}\left(-\frac{z}{2}, \frac{z}{2}\right) \psi\left(\frac{z}{2}\right)\right| p, \lambda\right\rangle\right|_{z^{0}=0, \vec{z}_{\perp}=\overrightarrow{0}_{\perp}},
$$

where $\mathscr{W}_{\mathrm{Q}}$ denotes a Wilson line. The unpolarized quasi-GPDs $H_{\mathrm{Q}(0 / 3)}$ and $E_{\mathrm{Q}(0 / 3)}$ are defined through the choice $\Gamma=\gamma^{0 / 3}$, the longitudinally polarized quasi-GPDs $\widetilde{H}_{\mathrm{Q}(0 / 3)}$ and $\widetilde{E}_{\mathrm{Q}(0 / 3)}$ through $\Gamma=\gamma^{0 / 3} \gamma_{5}$, and the transversely polarized quasi-GPDs $H_{T, \mathrm{Q}(0 / 3)}, E_{T, \mathrm{Q}(0 / 3)}, \widetilde{H}_{T, \mathrm{Q}(0 / 3)}$, and $\widetilde{E}_{T, \mathrm{Q}(0 / 3)}$ through $\Gamma=i \sigma^{j 0 / 3} \gamma_{5}$. See Ref. [3] for more details on the definition of quasi-GPDs.

Quasi-GPDs are functions of four kinematical variables: $x=k^{3} / P^{3}, \xi, t$ (or $\left|\vec{\Delta}_{\perp}\right|$ ) and $P^{3}=$ $\left(p^{3}+p^{\prime 3}\right) / 2$ (average z-component of hadron momentum). Note that the momentum fraction $x$ differs from $k^{+} / P^{+}$that appears for standard GPDs. Unlike the standard GPDs which extend from $-1<x<1$, the support for the quasi-GPDs is given by $-\infty<x<\infty$. We also use the quantity $\delta=\sqrt{1+\frac{M^{2}-t / 4}{\left(P^{3}\right)^{2}}}$ which shows up in the relation $P^{0}=\delta P^{3}$.

\section{Analytical Results of Quasi-GPDs in Scalar Diquark Model}

As an example, we quote here an expression of the unpolarized quasi-GPD $H_{\mathrm{Q}}$ defined with $\Gamma=\gamma^{0}$. Starting from an expression of the quasi-GPD correlator in the SDM (see Eq. (12) from Ref. [2]) and by using Gordon identities, one obtains

$$
H_{\mathrm{Q}(0)}\left(x, \xi, t ; P^{3}\right)=\frac{i g^{2} P^{3}}{(2 \pi)^{4}} \int d k^{0} d^{2} \vec{k}_{\perp} \frac{N_{H(0)}}{D_{\mathrm{GPD}}},
$$

where the numerator reads

$$
\begin{aligned}
N_{H(0)}= & \delta\left(k^{0}\right)^{2}-\frac{2}{P^{3}}\left[x\left(P^{3}\right)^{2}-m_{q} M-x \frac{t}{4}-\frac{1}{2} \delta \xi t \frac{\vec{k}_{\perp} \cdot \vec{\Delta}_{\perp}}{\vec{\Delta}_{\perp}^{2}}\right] k^{0} \\
& +\delta\left[x^{2}\left(P^{3}\right)^{2}+\vec{k}_{\perp}^{2}+m_{q}^{2}+(1-2 x) \frac{t}{4}-\delta \xi t \frac{\vec{k}_{\perp} \cdot \vec{\Delta}_{\perp}}{\vec{\Delta}_{\perp}^{2}}\right] .
\end{aligned}
$$

(The explicit expression for the denominator $D_{\mathrm{GPD}}$ can be found in Ref. [2]). While performing $\int d k^{0}$ via contour integration, one can verify that the position of the $k^{0}$-poles never switch half planes. This implies that all quasi-GPDs are continuous functions of $x$. (See Ref. [3] for results of all the quasi-GPDs corresponding to the twist- 2 standard GPDs.) 

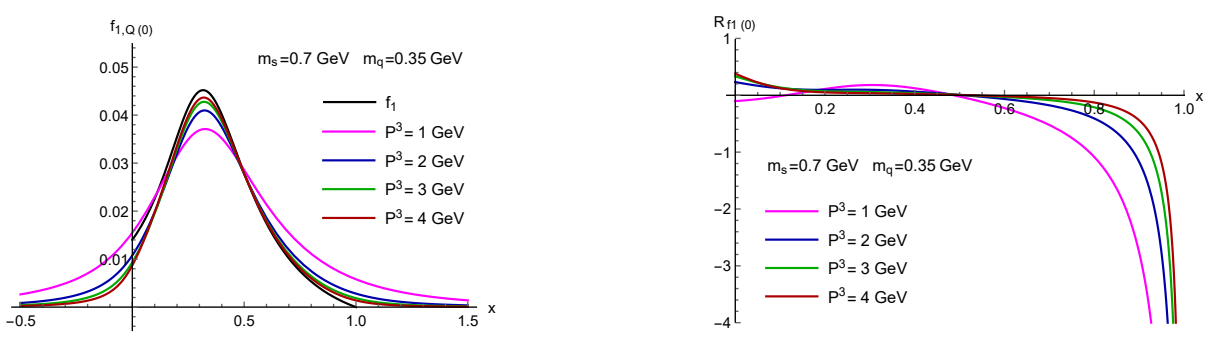

Figure 1: Left panel: Quasi-PDF $f_{1, \mathrm{Q}(0)}$ as a function of $x$ for different values of $P^{3}$. Black curve represents the standard PDF $f_{1}$. Right panel: Deviations at large $x$ made clearer through a relative-difference plot between $f_{1, \mathrm{Q}(0)}$ and $f_{1}$ as a function of $x$ for different values of $P^{3}$.
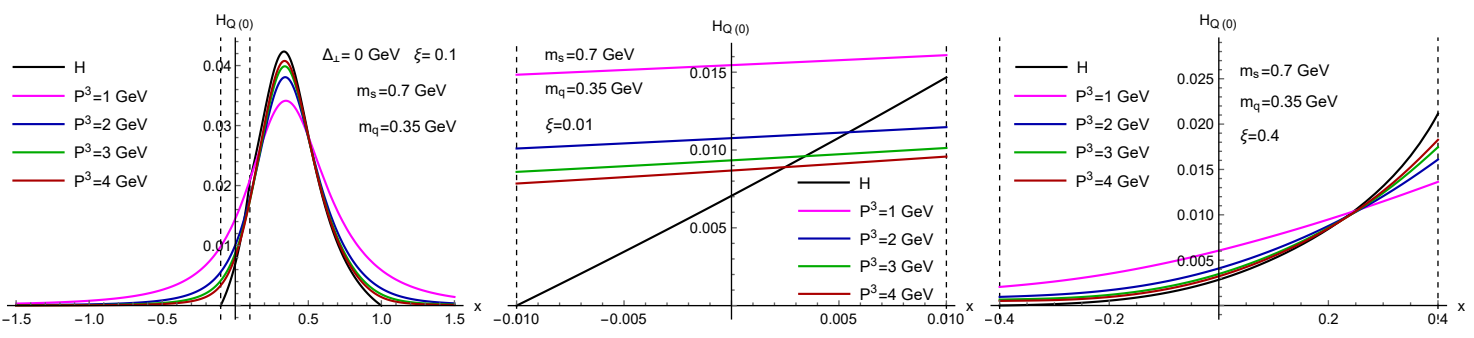

Figure 2: Left plot: Quasi-GPD $H_{\mathrm{Q}(0)}$ as a function of $x$ for $\xi=0.1$ and different values of $P^{3}$. Black curve represents the standard GPD $H$. The limits of the ERBL region are indicated by vertical dashed lines. Middle plot: $H_{\mathrm{Q}(0)}$ as a function of $x$ in the ERBL region for different values of $P^{3}$ and for $\xi=0.01$. Right plot: Result of $H_{\mathrm{Q}(0)}$ in the ERBL region for $\xi=0.4$.

\section{Numerical Results in Scalar Diquark Model}

Details regarding the choice of model parameters can be found in Ref. [2]. Our results discussed below are largely insensitive to variations of the model parameters [2,3]. Since most of the features exhibited by the quasi-GPDs become easier to grasp once one understands the qualitative behavior of the quasi-PDFs, we begin with discussing the PDFs first.

\subsection{Results for Quasi-PDFs}

The left plot in Fig.1 shows the unpolarized quasi-PDF $f_{1, \mathrm{Q}(0)}$, which is the forward limit of $H_{\mathrm{Q}(0)}$. For larger values of $P^{3}$, there is a good agreement between quasi and standard PDF over a wide range of $x$. However, considerable discrepancies appear as $x \rightarrow 0$ and $x \rightarrow 1$. As pointed out in [2], the discrepancy at small $x$ can be expected since, contrary to the continuous nature of the quasi-PDFs, the standard PDFs are discontinuous at $x=0$ in the SDM. The relative difference, defined as $R_{f 1(0)}\left(x ; P^{3}\right)=\frac{f_{1}(x)-f_{1, \mathrm{Q}(0)}\left(x ; P^{3}\right)}{f_{1}(x)}$, better illustrates the discrepancies at large $x$ and is shown in the right panel of Fig.1. In Ref. [3], we have pointed out that one may speculate about such substantial differences between the quasi and standard distributions at large $x$ due to huge discrepancies between the momentum fractions $k^{3} / P^{3}$ and $k^{+} / P^{+}$.

\subsection{Results for Quasi-GPDs}

The left plot in Fig. 2 shows the unpolarized $H_{\mathrm{Q}(0)}$ for $\xi=0.1$. For the skewness variable we 


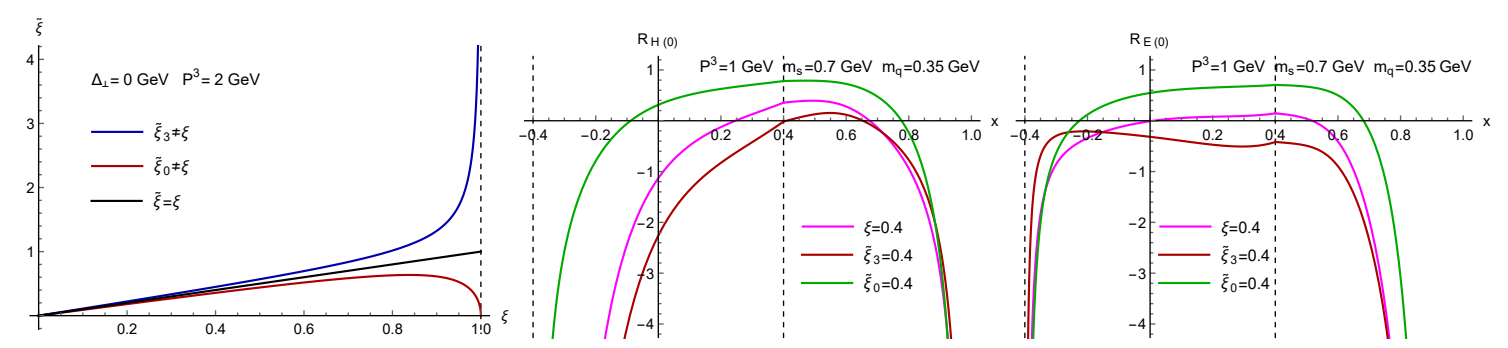

Figure 3: Left plot: Comparison of the skewness variables $\xi$, $\tilde{\xi}_{3}$ and $\tilde{\xi}_{0}$ for $P^{3}=2 \mathrm{GeV}$ and $\left|\vec{\Delta}_{\perp}\right|=0 \mathrm{GeV}$. Middle plot: Majority's trend reflected through a relative-difference plot between $H_{\mathrm{Q}(0)}$ and $H$ for three different definitions of the skewness variable. Right plot: Outlier's trend reflected through a relative-difference plot between $E_{\mathrm{Q}(0)}$ and $E$.

have explored the range $0.01 \leq \xi \leq 0.4$. The convergence problem at large $x$ persists for all the quasi-GPDs whether or not they have a forward counterpart. The middle and right plots in Fig. 2 shows $H_{\mathrm{Q}(0)}$ for just the ERBL region for $\xi=0.01$ and $\xi=0.4$, respectively. Generally, for small $\xi$ one finds significant deviations between the quasi-GPDs and the corresponding standard GPDs. This situation is the GPD counterpart of the problem for quasi-PDFs around $x=0$. Once $\xi$ is increased, we observe a (much) better agreement between quasi-GPDs and the standard GPDs for a large fraction of the ERBL region. This outcome suggests that lattice calculations could provide very valuable information in the ERBL region, provided that the skewness is not too small.

So far we have used the same skewness variable $\xi$ for both the standard GPDs and the quasiGPDs. However, for the quasi-GPDs one could in principle consider different variables to describe the longitudinal momentum transfer to the hadron. Examples in this context are $\tilde{\xi}_{3}=-\frac{\Delta^{3}}{2 P^{3}}$ and $\tilde{\xi}_{0}=-\frac{\Delta^{0}}{2 P^{0}}$. These "quasi skewness" variables are related to the standard skewness via a highertwist effect encoded in the model-independent relations $\tilde{\xi}_{3}=\delta \xi$ and $\tilde{\xi}_{0}=\frac{\xi}{\delta}$. The left plot of Fig. 3 shows considerable differences between $\xi, \tilde{\xi}_{3}$, and $\tilde{\xi}_{0}$ when $P^{3}$ is small. We explored the impact of the difference between these variables on the quasi-GPDs. Through the specific example of $H_{\mathrm{Q}(0)}$, the middle plot of Fig. 3 shows that ignoring the higher-twist effect and using $\tilde{\xi}_{3}$ leads to a better convergence of the majority of the quasi-GPDs for most of the DGLAP region. The only outliers in that regard are $E_{\mathrm{Q}(0)}, \widetilde{E}_{\mathrm{Q}(0 / 3)}$ and $E_{T, \mathrm{Q}(0)}$, where $E_{\mathrm{Q}(0)}$ is shown as a representative case in the right plot of Fig. 3. Also, using the variable $\tilde{\xi}_{0}$ typically gives poorer convergence for the quasi-GPDs. Our conclusions also hold for even larger values of $\xi$, where the numerical discrepancy between the three skewness variables increases further — see left plot of Fig. 3.

\section{Moments}

We first consider the lowest moment of the quasi-GPD $H_{\mathrm{Q}}$. Including a flavor index ' $q$ ' one finds the model-independent relation

$$
\int_{-1}^{1} d x H^{q}(x, \xi, t)=\int_{-\infty}^{\infty} d x \frac{1}{\delta} H_{\mathrm{Q}(0)}^{q}\left(x, \xi, t ; P^{3}\right)=\int_{-\infty}^{\infty} d x H_{\mathrm{Q}(3)}^{q}\left(x, \xi, t ; P^{3}\right)=F_{1}^{q}(t),
$$


where $F_{1}^{q}$ is the Dirac form factor for the quark flavor ' $q$ '. Despite the explicit dependence of the quasi-GPDs on $P^{3}$, it is remarkable that their lowest moments are independent of $P^{3}$. However one must divide half of the quasi-GPDs by the kinematical factor $\delta$ in order to arrive at this result. Since $\delta$ describes a higher-twist effect, including this factor is in principle a matter of taste. But the moment analysis suggests that taking into account $\delta$ like in (5.1) appears natural.

We now turn our attention to the second moment of quasi-GPDs considering again only the vector operator $\bar{\psi}^{q} \gamma^{\mu} \psi^{q}$. In close analogy to the celebrated expression for the second moment of $H+E$, namely $\int_{-1}^{1} d x x\left(H^{q}(x, \xi, t)+E^{q}(x, \xi, t)\right)=A^{q}(t)+B^{q}(t)$ where $A^{q}(0)+B^{q}(0)=J^{q}$ is the total angular momentum, one then finds for the quasi-GPDs

$$
\begin{aligned}
& \int_{-\infty}^{\infty} d x \frac{x}{\delta}\left(H_{\mathrm{Q}(0)}^{q}\left(x, \xi, t ; P^{3}\right)+E_{\mathrm{Q}(0)}^{q}\left(x, \xi, t ; P^{3}\right)\right)=\frac{\left(\delta^{2}+1\right)}{2}\left(A^{q}(t)+B^{q}(t)\right)+\frac{\left(\delta^{2}-1\right)}{2} D^{q}(t),(5.2) \\
& \int_{-\infty}^{\infty} d x x\left(H_{\mathrm{Q}(3)}^{q}\left(x, \xi, t ; P^{3}\right)+E_{\mathrm{Q}(3)}^{q}\left(x, \xi, t ; P^{3}\right)\right)=A^{q}(t)+B^{q}(t)
\end{aligned}
$$

Note that in Eq. (5.2) the form factor $D^{q}$ of the anti-symmetric part of the energy momentum tensor enters. One can conclude that the second moment of $H_{\mathrm{Q}(3)}+E_{\mathrm{Q}(3)}$ is directly related to the angular momentum of quarks, while for $H_{\mathrm{Q}(0)}+E_{\mathrm{Q}(0)}$ this relation contains a higher-twist "contamination." The model-independent expressions for the moments of the quasi distributions may be useful for studying the systematic uncertainties of results from lattice $\mathrm{QCD}$, especially due to the fact that the $P^{3}$-dependence of the moments is either computable or nonexistent.

\section{Summary}

We have studied twist-2 GPDs through parton quasi-distributions in the SDM. Our analytical expressions for the quasi-GPDs reduce to the respective standard GPDs for $P^{3} \rightarrow \infty$, which further validates quasi-GPDs as viable tools for getting information about standard GPDs. For finite $P^{3}$ and large $\xi$, quasi-GPDs agree well with the standard distributions in the ERBL region. This agreement gets poorer if $\xi$ gets smaller. We have provided a model-independent analysis of moments of quasiGPDs including the relation to Ji's spin sum rule. The moment analysis may assist in the study of systematic uncertainties in lattice QCD.

\section{References}

[1] X. Ji, Parton physics on a Euclidean lattice, Phys. Rev. Lett. 110, 262002 (2013).

[2] S. Bhattacharya, C. Cocuzza and A. Metz, Generalized quasi parton distributions in a diquark spectator model, Phys. Lett. B 788, 453 (2019).

[3] S. Bhattacharya, C. Cocuzza and A. Metz, Exploring twist-2 GPDs through quasi-distributions in a diquark spectator model, arXiv:1903.05721 [hep-ph].

[4] M. Diehl, Generalized parton distributions, Phys. Rept. 388, 41 (2003). 